\title{
Targeting Membrane Lipid a Potential Cancer Cure?
}

\author{
Loh Teng-Hern Tan', Kok-Gan Chan², Priyia Pusparajah ${ }^{3}$, Wai-Leng Lee, \\ Lay-Hong Chuah', Tahir Mehmood Khan ${ }^{1,5}$, Learn-Han Lee ${ }^{1,6 *}$ and Bey-Hing Goh 1,6*
}

${ }^{1}$ Novel Bacteria and Drug Discovery Research Group, School of Pharmacy, Monash University Malaysia, Bandar Sunway, Malaysia, ${ }^{2}$ Division of Genetics and Molecular Biology, Institute of Biological Sciences, Faculty of Science, University of Malaya, Kuala Lumpur, Malaysia, ${ }^{3}$ Biomedical Research Laboratory, Jeffrey Cheah School of Medicine and Health Sciences, Monash University Malaysia, Bandar Sunway, Malaysia, ${ }^{4}$ School of Science, Monash University Malaysia, Selangor, Malaysia, ${ }^{5}$ Department of Pharmacy, Abasyn University Peshawar, Peshawar, Pakistan, ${ }^{6}$ Center of Health Outcomes Research and Therapeutic Safety, School of Pharmaceutical Sciences, University of Phayao, Phayao, Thailand

Cancer mortality and morbidity is projected to increase significantly over the next few decades. Current chemotherapeutic strategies have significant limitations, and there is great interest in seeking novel therapies which are capable of specifically targeting cancer cells. Given that fundamental differences exist between the cellular membranes of healthy cells and tumor cells, novel therapies based on targeting membrane lipids in cancer cells is a promising approach that deserves attention in the field of anticancer drug development. Phosphatidylethanolamine (PE), a lipid membrane component which exists only in the inner leaflet of cell membrane under normal circumstances, has increased surface representation on the outer membrane of tumor cells with disrupted membrane asymmetry. PE thus represents a potential chemotherapeutic target as the higher exposure of PE on the membrane surface of cancer cells. This feature as well as a high degree of expression of PE on endothelial cells in tumor vasculature, makes PE an attractive molecular target for future cancer interventions. There have already been several small molecules and membrane-active peptides identified which bind specifically to the PE molecules on the cancer cell membrane, subsequently inducing membrane disruption leading to cell lysis. This approach opens up a new front in the battle against cancer, and is of particular interest as it may be a strategy that may be prove effective against tumors that respond poorly to current chemotherapeutic agents. We aim to highlight the evidence suggesting that PE is a strong candidate to be explored as a potential molecular target for membrane targeted novel anticancer therapy.

Keywords: phosphatidylethanolamine, phospholipid bilayer, targeted drug, anticancer, membrane permeabilization

\section{INTRODUCTION}

Cancer represents a major health concern globally. In 2012, cancer was responsible for 8 million deaths, with an estimated 14 million new cases diagnosed. Recent decades have seen advances in the diagnosis and treatment of cancer, but the overall reduction in cancer mortality is still limited; and even more worryingly, cancer-related cases and deaths are projected to rise by at least $70 \%$ by 2030 (Ferlay et al., 2015; Antoni et al., 2016). Even if patients do successfully overcome cancer through the current standard mainstays of treatment - namely chemotherapy and radiotherapy - the risk 
of reoccurrence of the disease is a major concern. Currently, the main issues of concern relating to the currently available therapeutic options are low therapeutic indices and a broad spectrum of adverse effects (MacDonald, 2009). The key issues underlying problems of potential toxicity as well as drug resistance are attributed to the lack of specificity of existing therapies toward tumor cells (Chari, 2007). Drug resistance can be intrinsic because of variations between individual patients and also due to the genetic differences in tumors (Gottesman et al., 2002). Increasing evidence has demonstrated various mechanisms by which cancer cells may acquire resistance such as drug-detoxifying mechanisms, expression of one or more energydependent transporters that extrude anticancer agent from the cells before interaction with intracellular targets take place, or acquiring the ability to evade drug induced apoptosis (Gottesman et al., 2002; Perez-Tomas, 2006; Gillet and Gottesman, 2010; Batist et al., 2011). Taken together, there is a mandate to develop innovative therapeutic strategies to overcome the limitations of current therapy, subsequently lowering the ever-increasing cancer related mortality and morbidity.

In the late 1800s, bacteriologist Paul Ehrlich first conceptualized the 'magic bullet', a specifically synthesized drug that would target a specific microorganism while not harming any other cells in the organism. This concept has now evolved into a more general field aiming to develop targeted drug delivery strategies (Imai and Takaoka, 2006) to treat various diseases including cancer and inflammatory diseases (Allen and Cullis, 2004). Currently, these efforts have resulted in the development of a full range of powerful molecular therapeutics including monocompounds with targeting properties or a cocktail of therapeutic compounds which may comprise fusion proteins and nano/microparticles. The clinical success of targeted drug delivery is highly dependent on its delivery vehicle and its targets. In cancer treatment, a number of targeting functions have been discovered such as tumor-antigen recognizing antibodies (Kirpotin et al., 2006), ligands for receptors (Pastorino et al., 2006) and RNA aptamers (Farokhzad et al., 2006) against antigens which are expressed on the surface of tumor cells. However, actually utilizing this discovery in developing targeted drug delivery systems is limited by virtue of the lack of an ubiquitously expressed tumor-specific antigen or receptor on various cancer cells. Also, the geno- and phenotypic heterogeneity of individual tumors (Marusyk et al., 2012) and the co-existence of drug-susceptible and drug-resistant clones (Greaves and Maley, 2012) further complicates targeted therapy.

In addition to the concerns involving treatment of localized cancers, to date there has also been a lack of success in developing an effective treatment for disseminated cancer. Over the last few decades, it has been shown that the overall contribution of curative and adjuvant cytotoxic chemotherapy has not resulted in substantial improvement of treatment outcomes for most cancer types (Bailar and Gornik, 1997; Morgan et al., 2004; Ashdown et al., 2015). Thus, there is a clearly an urgent need to pursue ground breaking, innovative ideas in developing alternative chemotherapeutics that act specifically on clearly defined biomarkers which are generally expressed within certain tumor types in order to improve cancer cell selectivity of chemotherapeutic agents. Considerable effort has been devoted to developing novel targeted anticancer strategies such as specific agents able to inhibit cancer cell proliferation (Parenti et al., 2014), promote cell cycle regulation (Gabrielli et al., 2012) and induce apoptosis or autophagy (Amaravadi et al., 2011). However, a promising new approach targeting the cancer cell membrane seems to have been underexplored thus far. This approach focuses on destroying cancer cells by damaging their cell membranes instead of binding to specific receptors. Here we aim to highlight the prospect of phosphatidylethanolamine (PE), an aminophospholipid on the cell membrane, as an attractive target for anti-cancer therapy. A schematic diagram depicting the potential of PE as molecular target for peptides and small molecules exhibiting anticancer properties (Figure 1) has been included.

\section{MEMBRANE TARGETED THERAPY}

Generally, most marketed drugs elicit their effects via direct interaction with proteins (Melnikova and Golden, 2004; Adrian et al., 2007); in fact, many new molecular entities are developed for clinical use based on the previously elucidated structure of their intended target proteins (Prathipati et al., 2007). However, as increasing evidence emerges regarding the role of membrane lipids in the regulation of numerous cellular functions and activity of membrane proteins, membrane lipids have emerged as an alternative molecular target (Lee, 2004, 2011). Given that many cellular functions occur in or around the cell membrane, alterations in the types and/or levels of membrane lipids may have implications for many human pathologies, hence it was proposed that therapies based on the regulation of membrane lipids structure could be developed to control the molecular events that are relevant to the various pathological states (Escribá, 2006). This has led to membrane-lipid therapy, which aims to reverse the pathological state of the cell membrane by using drugs that influence the membrane's lipid organization via the principle of structure-function, subsequently inducing changes in cell signaling and gene expression (Escribá, 2006).

The development of cell membrane targeted cancer therapy is made possible by the fact that there are fundamental differences between the cell membrane composition of normal and cancer cells. Non-cancer cells usually exhibit a more neutral total membrane charge attributed to the presence of zwitterionic phospholipids such as phosphatidylcholine and sphingomyelin while phosphatidylserine (PS) and PE (are) located in the inner leaflet of plasma membrane (Bevers et al., 1996). The asymmetrical distribution of the phospholipids is maintained by a group of P-type ATPases, aminophospholipid translocases that use ATP hydrolysis to flip PS and PE from the external to cytosolic leaflet (Chen et al., 1999). Unlike normal cells, apoptotic cells and cancer cells lose their capacity to maintain PS asymmetry, leading to the exposure of this lipid at the cell's outer membrane leaflet. PS is one of the molecules that has gained interest in various clinical disciplines, particularly in the development of non-invasive imaging technology to support diagnosis and evaluation of treatment efficacy for various 


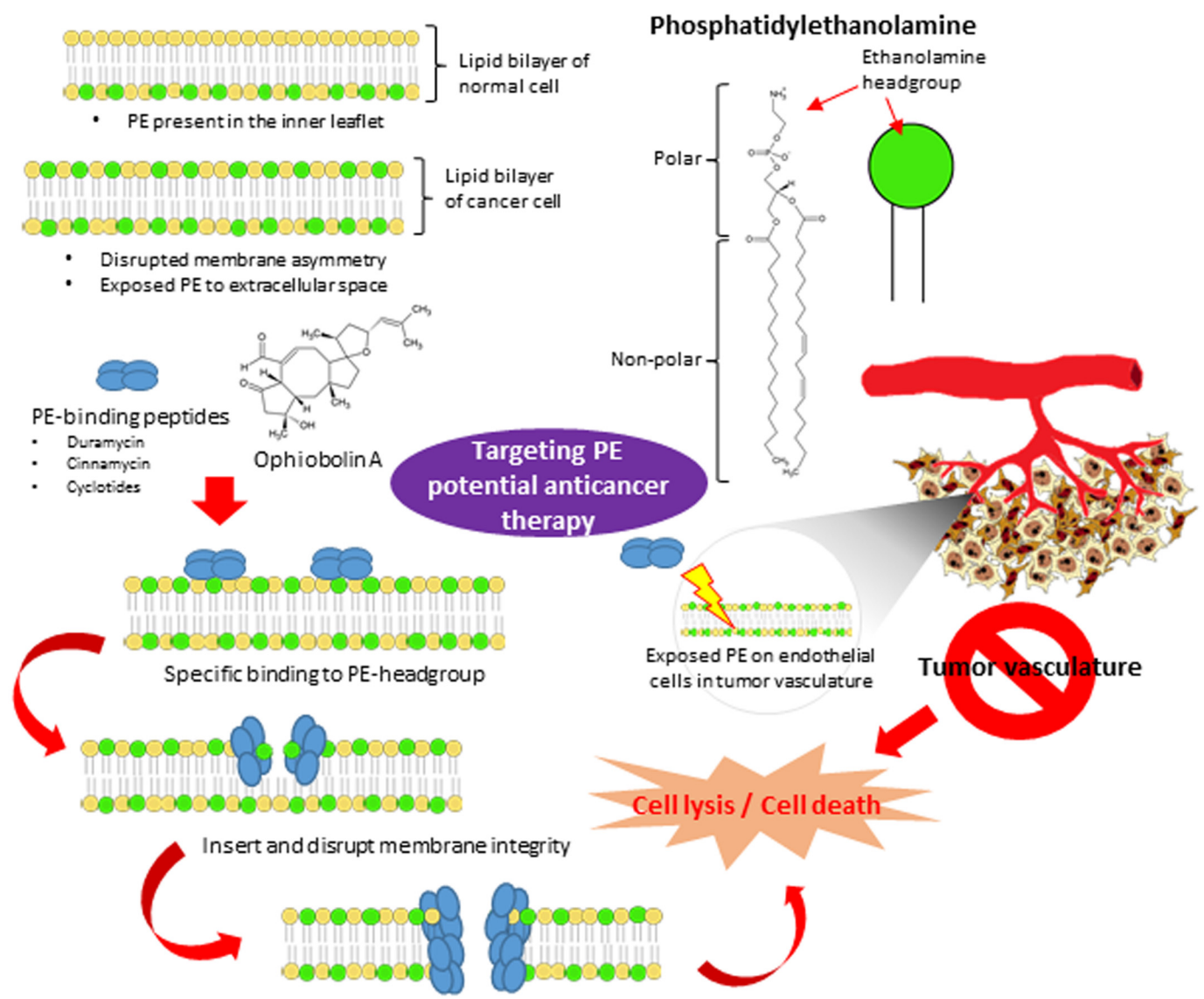

Pore formation and membrane permeabilization

FIGURE 1 | The potential of PE as the novel molecular therapeutic target in anticancer treatment. The plasma membrane of normal cells is characterized by an asymmetric distribution of various phospholipids over two membrane leaflet. PE resides in the inner leaflet facing the cytosol. The disrupted membrane asymmetry of cancer cell with exposed PE to extracellular space serves as a molecular target for anticancer therapy. PE-binding peptides and small molecules (ophiobolin A) bind specifically to the ethanolamine headgroup of PE. Upon binding, they disrupt and induce membrane destabilization via insertion into the lipid bilayer. They also may induce membrane permeabilization via pore formation, subsequently lead to cell lysis and cell death. Furthermore, PE is also highly exposed on endothelium cells in tumor vasculature. Targeting PE on endothelial cells in tumor vasculature could be a potential anti-angiogenesis approach to inhibit tumor development.

diseases such as cancer and cardiovascular disease (Kenis and Reutelingsperger, 2009). Furthermore, PS also represents an attractive target for targeted drug delivery strategies that aim to treat disease effectively with minimal detrimental side-effects. Due to the limited PE-specific probes available to facilitate the study of behavior of cell surface PE in living systems, the functional significance of PE is less well-documented as compared to that of PS. To date, duramycin and cinnamycin are the only two well-known PE-binding peptides that have assisted in unraveling many important biological functions of PE (Zhao, 2011).

\section{PHOSPHATIDYLETHANOLAMINE AS AN ANTI-CANCER TARGET}

Phosphatidylethanolamine is the second most abundant phospholipid on mammalian cellular membranes, accounting for approximately $20 \%$ of the total phospholipids (Spector and Yorek, 1985). Generally, PE is found predominantly in the inner leaflet of the plasma membrane of viable mammalian cells. In addition, $\mathrm{PE}$ is also abundant in the inner membrane of mitochondria (Vance, 2008). Besides functioning as a membrane structural element, the evidence suggests that PE participates in many important pathophysiological cellular processes (Vance, 2008; Calzada et al., 2016). It has been demonstrated that translocation and redistribution of $\mathrm{PE}$ occurs during a number of distinct biological events: (1) cell division where it is externalized at the cleavage furrow (Emoto et al., 1996); (2) cell death (for which exposure of PE to extracellular environment is a molecular marker) (Emoto et al., 1997); (3) anticoagulant mechanism where PE acts as a cofactor for protein C (Tian et al., 2006). Given the diversity in its biological roles, PE represents a distinct molecular target among aminophospholipids and other membrane phospholipid components. 
The development of PE-specific probes derived from duramycin and cinnamycin has made possible the recent discoveries of many biological functions of PE in living systems. Duramycin is a highly specific PE-binding peptide produced by Streptoverticillium cinnamoneus and is closely related to cinnamycin (Ro09-0198) which is produced by Streptomyces sp. (Iwamoto et al., 2007). Both duramycin and cinnamycin are lantibiotics, bacteriocins which are characterized by the presence of a high proportion of unusual thioether amino acids (lanthionine and methyllanthionine). Lantibiotics are toxic toward many Gram-positive bacteria, but the toxic effects of cinnamycin are not limited to bacterial cells. Duramycin and cinnamycin were also found to display a number of effects on eukaryotic cells, such as induction of hemolysis (Choung et al., 1988a), inhibition of phospholipase A2 or interference with prostaglandin and leukotriene biosynthesis (Märki et al., 1991). Most of these effects described can be explained by the specific binding of these lantibiotics to PE (Choung et al., 1988b; Hosoda et al., 1996).

Both duramycin and cinnamycin are unique in that they bind to PE located in the inner layer of the plasma membrane in mammalian cells. They bind with high affinity to the head group of $\mathrm{PE}$ at a molar ratio of 1:1 with a dissociation constant in the nanomolar range (Zhao et al., 2008). Given that they specifically bind to $\mathrm{PE}$, they have been employed to study the distribution and metabolism of $\mathrm{PE}$ in different biological systems. The binding of cinnamycin to PE was found to induce transbilayer phospholipid movement of target cells in a PE-dependent manner (Makino et al., 2003). This study demonstrated that the binding of cinnamycin induces membrane reorganization that eventually leads to cell death (Makino et al., 2003). In addition to inducing transbilayer lipid movement, there is preferential binding of both duramycin and cinnamycin to areas of membrane with high curvature and further binding of the peptides is promoted via membrane tubulation (Iwamoto et al., 2007).

In addition to duramycin and cinnamycin, cyclotides represent a new lipid-binding protein family of cyclic peptides which exert their biological activities by acting on cell membrane, particularly by binding to phospholipids containing PE headgroups. Studies have shown that the cyclotides interact and bind specifically with membranes containing PE-phospholipids as compared to other lipids lacking PEphospholipids and their binding was followed by insertion that subsequently led to membrane disruption (Henriques et al., 2011, 2012; Troeira Henriques et al., 2014). Wang et al. (2012) proposed that the binding of cyclotides to cell membrane surface via interaction with PE-lipid headgroup resulted in formation of pores based on barrel-stave or toroidal pore models when a threshold concentration is exceeded. The toxicity of cyclotides were shown to be dependent on PE exposure at the membrane surface. Although cyclotides were found to be equally toxic toward cancerous and non-cancerous cell, cyclotides can recognize cancer cells more efficiently than non-cancerous cells at non-permeabilising concentrations (Troeira Henriques et al., 2014). Hence, the higher proportion of exposed $\mathrm{PE}$ on the outer membrane of cancer cell presents a potential molecular target for those membraneactive peptides to exert their cytotoxic effects. In addition, $\mathrm{PE}$ was found to play a role in enhancing the susceptibility of the membrane to permeabilization by bound peptides and also facilitating the formation of larger transmembrane pores (Leite et al., 2015). These findings open up new avenues toward the development of novel chemotherapeutic peptides that specifically recognize $\mathrm{PE}$ phospholipid components on cancer cells and induce membrane disruption upon binding, leading to membrane permeabilization via pore formation and eventually cell death.

Despite numerous successful examples of membrane active peptides in in vivo studies, to date none of them have made it into the pharmaceutical market. Potential issues including poor bioavailability and potential toxicity of peptides are hampering the progress and development of peptides as novel anticancer agents. Furthermore, peptide degradation due to serum proteases is also a major challenge limiting the stability and half-life of these molecules (Adessi and Soto, 2002).

A recent study has found that a natural product, ophiobolin A (OPA) exerts its cytotoxicity toward cancer cells by forming a pyrrole-containing covalent cytotoxic adduct with the ethanolamine head group of PE, subsequently leading to lipid bilayer destabilization (Chidley et al., 2016). OPA is a plant toxin isolated from pathogenic fungi of the Bipolaris genus that exhibits cytotoxicity at nanomolar concentrations against a range of cancer cell lines. Basically, the study performed an unbiased genome-wide approach to identify the molecular target of OPA through insertional mutagenesis of near-haploid cell line KBM7 using retroviral gene trap approach and demonstrated that OPA forms PE-OPA adducts associated with membrane disruption and eventual cell death (Chidley et al., 2016). This discovery highlighted the potential importance of PE as the molecular target for small molecules which provides further impetus to pursue targeting $\mathrm{PE}$ as a novel chemotherapeutic approach, particularly against cancer types which are known to respond poorly to current chemotherapy.

Additional information from a recent study revealed that $\mathrm{PE}$ is highly exposed on tumor vascular endothelium, suggesting that it may serve as a marker for imaging tumor vasculature and also drug targeting (Stafford and Thorpe, 2011). Targeting the angiogenesis-driven sprouting of new vessels has seen a revolution in anti-cancer drug development in the past decade. Angiogenesis and tumor vascularization are crucial to the growth and metastasis of cancers (Folkman, 2002; Berretta et al., 2016). The observation that tumors cannot grow beyond a size of approximately $2 \mathrm{~mm}^{3}$ without the support of neovascularization (Folkman, 1971) has led to clinical development of a large number of angiogenesis-inhibiting agents that act on a number of potential targets, including the matrix-metalloproteinases, tissue plasminogen activator (Pepper, 2001), angiogenic cytokines and vascular endothelial cells (Dvorak, 2002) participating in angiogenesis. Therefore, the selective exposure of PE on tumor vasculature may present an opportunity for selective therapeutic intervention, whereby specific PE-binding peptides or antibodies can be employed to direct or deliver the bound endothelial cell-damaging agents to the site of action. 


\section{CONCLUSION AND FUTURE DIRECTIONS}

To date, the success of cancer therapies is limited by their severe side effects and also the problems of drug resistance. These issues make it vital to explore potential molecular targets for anticancer therapy like PE which have strong potential for translation into future clinical applications. A number of membrane-active peptides and small molecules have been shown to bind PE specifically, subsequently eliciting membrane disruption. The ability of those molecules to recognize cancer cells is PE dependent, suggesting that PE is an efficient means of targeting cancers. This notion is strengthened further by the finding that the levels of $\mathrm{PE}$ exposure on the outer membrane leaflet is particularly high on cell surfaces exposed to stress conditions associated with tumor microenvironments. However, optimization and modification of peptides are required to enhance their selective interaction with their target on the cell membrane as well as reducing toxicity and development of drug resistance. The knowledge of these PE-binding peptides and small molecules has the potential to assist in the design of novel drugs based on their molecular scaffold, perhaps allowing development of molecules capable of targeting specific cell types. Based on the concept of membrane disruption via binding of $\mathrm{PE}$,

\section{REFERENCES}

Adessi, C., and Soto, C. (2002). Converting a peptide into a drug: strategies to improve stability and bioavailability. Curr. Med. Chem. 9, 963-978. doi: 10. 2174/0929867024606731

Adrian, G. L., Marcel, V., Robert, B. G., and Richard, T. (2007). A comparison of physicochemical property profiles of marketed oral drugs and orally bioavailable anti-cancer protein kinase inhibitors in clinical development. Curr. Top. Med. Chem. 7, 1408-1422. doi: 10.2174/156802607781 696819

Allen, T. M., and Cullis, P. R. (2004). Drug delivery systems: entering the mainstream. Science 303, 1818-1822. doi: 10.1126/science.1095833

Amaravadi, R. K., Lippincott-Schwartz, J., Yin, X.-M., Weiss, W. A., Takebe, N., Timmer, W., et al. (2011). Principles and current strategies for targeting autophagy for cancer treatment. Clin. Cancer Res. 17, 654-666. doi: 10.1158/ 1078-0432.ccr-10-2634

Antoni, S., Soerjomataram, I., Møller, B., Bray, F., and Ferlay, J. (2016). An assessment of GLOBOCAN methods for deriving national estimates of cancer incidence. Bull. World Health Organ. 94, 174-184. doi: 10.2471/blt.15.164384

Ashdown, M. L., Robinson, A. P., Yatomi-Clarke, S. L., Ashdown, M. L., Allison, A., Abbott, D., et al. (2015). Chemotherapy for late-stage cancer patients: meta-analysis of complete response rates. F1000Res. 4:232. doi: 10 . 12688/f1000research.6760.1

Bailar, J. C., and Gornik, H. L. (1997). Cancer undefeated. N. Engl. J. Med. 336, 1569-1574. doi: 10.1056/nejm199705293362206

Batist, G., Wu, J. H., Spatz, A., Miller, W. H., Cocolakis, E., Rousseau, C., et al. (2011). Resistance to cancer treatment: the role of somatic genetic events and the challenges for targeted therapies. Front. Pharmacol. 2:59. doi: 10.3389/fphar. 2011.00059

Berretta, M., Rinaldi, L., Di Benedetto, F., Lleshi, A., De Re, V., Facchini, G., et al. (2016). Angiogenesis inhibitors for the treatment of hepatocellular carcinoma. Front. Pharmcol. 7:428. doi: 10.3389/fphar.2016.00428

Bevers, E., Comfurius, P., and Zwaal, R. (1996). Regulatory mechanisms in maintenance and modulation of transmembrane lipid asymmetry: pathophysiological implications. Lupus 5, 480-487. doi: 10.1177/ 096120339800700228 suitable assays employing high-throughput screening strategy can be used to identify novel PE-binding molecules from large libraries of drug candidates before optimization in terms of their potency, selectivity, physicochemical, pharmacokinetic, and toxicity properties.

\section{AUTHOR CONTRIBUTIONS}

LT contributed to the literature database search and writing of the manuscript. L-HL, K-GC, W-LL, L-HC, TK, PP, and B-HG contributed vital insights and proofread the writing. The research topic was conceptualized by B-HG.

\section{ACKNOWLEDGMENTS}

This work was supported by PVC Award Grant (Project No. PVC-ECR-2016), External Industry Grant (Biotek Abadi - Vote No. GBA-808813), MOSTI eScience funds (Project No. 02-0210-SF0215 and 06-02-10-SF0300), Fundamental Research Grant Scheme (FRGS/1/2014/SKK01/MUSM/03/2) and University of Malaya for High Impact Research Grant (UM-MOHE HIR Nature Microbiome Grant No. H-50001-A000027 and No. A000001-50001) and PG136-2016A.

Calzada, E., Onguka, O., and Claypool, S. M. (2016). Chapter Twophosphatidylethanolamine metabolism in health and disease. Int. Rev. Cell Mol. Biol. 321, 29-88. doi: 10.1016/bs.ircmb.2015.10.001

Chari, R. V. (2007). Targeted cancer therapy: conferring specificity to cytotoxic drugs. Acc. Chem. Res. 41, 98-107. doi: 10.1021/ar700108g

Chen, C.-Y., Ingram, M. F., Rosal, P. H., and Graham, T. R. (1999). Role for Drs2p, a P-type ATPase and potential aminophospholipid translocase, in yeast late Golgi function. J. Cell Biol. 147, 1223-1236. doi: 10.1083/jcb.147.6.1223

Chidley, C., Trauger, S. A., Birsoy, K., and O'shea, E. K. (2016). The anticancer natural product ophiobolin A induces cytotoxicity by covalent modification of phosphatidylethanolamine. Elife 5:e14601. doi: 10.7554/elife.14601

Choung, S.-Y., Kobayashi, T., Inoue, J.-I., Takemoto, K., Ishitsuka, H., and Inoue, K. (1988a). Hemolytic activity of a cyclic peptide Ro09-0198 isolated from Streptoverticillium. Biochm. Biophys. Acta 940, 171-179. doi: 10.1016/ 0005-2736(88)90192-7

Choung, S.-Y., Kobayashi, T., Takemoto, K., Ishitsuka, H., and Inoue, K. (1988b). Interaction of a cyclic peptide, Ro09-0198, with phosphatidylethanolamine in liposomal membranes. Biochm. Biophys. Acta 940, 180-187. doi: 10.1016/00052736(88)90193-9

Dvorak, H. F. (2002). Vascular permeability factor/vascular endothelial growth factor: a critical cytokine in tumor angiogenesis and a potential target for diagnosis and therapy. J. Clin. Oncol. 20, 4368-4380. doi: 10.1200/jco.2002. 10.088

Emoto, K., Kobayashi, T., Yamaji, A., Aizawa, H., Yahara, I., Inoue, K., et al. (1996). Redistribution of phosphatidylethanolamine at the cleavage furrow of dividing cells during cytokinesis. Proc. Natl. Acad. Sci. U.S.A. 93, 12867-12872. doi: 10.1073/pnas.93.23.12867

Emoto, K., Toyama-Sorimachi, N., Karasuyama, H., Inoue, K., and Umeda, M. (1997). Exposure of phosphatidylethanolamine on the surface of apoptotic cells. Exp. Cell Res. 232, 430-434. doi: 10.1006/excr.1997.3521

Escribá, P. V. (2006). Membrane-lipid therapy: a new approach in molecular medicine. Trends Mol. Med. 12, 34-43. doi: 10.1016/j.molmed.2005.11.004

Farokhzad, O. C., Cheng, J., Teply, B. A., Sherifi, I., Jon, S., Kantoff, P. W., et al. (2006). Targeted nanoparticle-aptamer bioconjugates for cancer chemotherapy in vivo. Proc. Natl. Acad. Sci. U.S.A. 103, 6315-6320. doi: 10.3410/f.1032208. 373127 
Ferlay, J., Soerjomataram, I., Dikshit, R., Eser, S., Mathers, C., Rebelo, M., et al. (2015). Cancer incidence and mortality worldwide: sources, methods and major patterns in GLOBOCAN 2012. Int. J. Cancer 136, E359-E386. doi: 10.1002/ijc. 29210

Folkman, J. (1971). Tumor angiogenesis: therapeutic implications. N. Engl. J. Med. 285, 1182-1186. doi: 10.1056/nejm197111182852108

Folkman, J. (2002). Role of angiogenesis in tumor growth and metastasis. Semin. Oncol. 29, 15-18. doi: 10.1016/s0093-7754(02)70065-1

Gabrielli, B., Brooks, K., and Pavey, S. (2012). Defective cell cycle checkpoints as targets for anti-cancer therapies. Front. Pharmacol. 3:9. doi: 10.3389/fphar.2012. 00009

Gillet, J.-P., and Gottesman, M. M. (2010). Mechanisms of multidrug resistance in cancer. Methods Mol. Biol. 596, 47-76. doi: 10.1007/978-1-60761-416-6_4

Gottesman, M. M., Fojo, T., and Bates, S. E. (2002). Multidrug resistance in cancer: role of ATP-dependent transporters. Nat. Rev. Cancer 2, 48-58. doi: 10.1038/ nrc706

Greaves, M., and Maley, C. C. (2012). Clonal evolution in cancer. Nature 481, 306-313. doi: 10.1038/nature10762

Henriques, S. T., Huang, Y.-H., Castanho, M. A., Bagatolli, L. A., Sonza, S., Tachedjian, G., et al. (2012). Phosphatidylethanolamine binding is a conserved feature of cyclotide-membrane interactions. J. Biol. Chem. 287, 33629-33643. doi: 10.1074/jbc.m112.372011

Henriques, S. T., Huang, Y.-H., Rosengren, K. J., Franquelim, H. G., Carvalho, F. A., Johnson, A., et al. (2011). Decoding the membrane activity of the cyclotide kalata B1 the importance of phosphatidylethanolamine phospholipids and lipid organization on hemolytic and anti-HIV activities. J. Biol. Chem. 286, 24231-24241. doi: 10.1074/jbc.m111.253393

Hosoda, K., Ohya, M., Kohno, T., Maeda, T., Endo, S., and Wakamatsu, K. (1996). Structure determination of an immunopotentiator peptide, cinnamycin, complexed with lysophosphatidylethanolamine by 1H-NMR. J. Biochem. 119, 226-230. doi: 10.1093/oxfordjournals.jbchem.a021226

Imai, K., and Takaoka, A. (2006). Comparing antibody and small-molecule therapies for cancer. Nat. Rev. Cancer 6, 714-727. doi: 10.1038/nrc1913

Iwamoto, K., Hayakawa, T., Murate, M., Makino, A., Ito, K., Fujisawa, T., et al. (2007). Curvature-dependent recognition of ethanolamine phospholipids by duramycin and cinnamycin. Biophys. J. 93, 1608-1619. doi: 10.1016/j. chemphyslip.2007.06.067

Kenis, H., and Reutelingsperger, C. (2009). Targeting phosphatidylserine in anti-cancer therapy. Curr. Pharm. Des. 15, 2719-2723. doi: 10.2174/ 138161209788923903

Kirpotin, D. B., Drummond, D. C., Shao, Y., Shalaby, M. R., Hong, K., Nielsen, U. B., et al. (2006). Antibody targeting of long-circulating lipidic nanoparticles does not increase tumor localization but does increase internalization in animal models. Cancer Res. 66, 6732-6740. doi: 10.1158/0008-5472.can-05-4199

Lee, A. G. (2004). How lipids affect the activities of integral membrane proteins. Biochim. Biophys. Acta 1666, 62-87. doi: 10.1016/j.bbamem.2004.05.012

Lee, A. G. (2011). Lipid-protein interactions. Biochem. Soc. Trans. 39, 761-766. doi: 10.1042/bst0390761

Leite, N. B., Aufderhorst-Roberts, A., Palma, M. S., Connell, S. D., Neto, J. R., and Beales, P. A. (2015). PE and PS lipids synergistically enhance membrane poration by a peptide with anticancer properties. Biophys. J. 109, 936-947. doi: 10.1016/j.bpj.2015.07.033

MacDonald, V. (2009). Chemotherapy: managing side effects and safe handling. Can. Vet. J. 50, 665-668.

Makino, A., Baba, T., Fujimoto, K., Iwamoto, K., Yano, Y., Terada, N., et al. (2003). Cinnamycin (Ro 09-0198) promotes cell binding and toxicity by inducing transbilayer lipid movement. J. Biol. Chem. 278, 3204-3209. doi: 10.1074/jbc. $\mathrm{m} 210347200$

Märki, F., Hänni, E., Fredenhagen, A., and Van Oostrum, J. (1991). Mode of action of the lanthionine-containing peptide antibiotics duramycin, duramycin $\mathrm{B}$ and $\mathrm{C}$, and cinnamycin as indirect inhibitors of phospholipase A2. Biochem. Pharmacol. 42, 2027-2035. doi: 10.1016/0006-2952(91)90604-4

Marusyk, A., Almendro, V., and Polyak, K. (2012). Intra-tumour heterogeneity: a looking glass for cancer? Nat. Rev. Cancer 12, 323-334. doi: 10.1038/nrc3261
Melnikova, I., and Golden, J. (2004). Targeting protein kinases. Nat. Rev. Drug Discov. 3, 993-994. doi: 10.1038/nrd1600

Morgan, G., Ward, R., and Barton, M. (2004). The contribution of cytotoxic chemotherapy to 5-year survival in adult malignancies. Clin. Oncol. 16, 549-560. doi: 10.1016/j.clon.2004.06.007

Parenti, R., Cardile, V., Graziano, A. C. E., Parenti, C., Venuti, A., Bertuccio, M. P., et al. (2014). Wilms' tumor gene 1 (WT1) silencing inhibits proliferation of malignant peripheral nerve sheath tumor sNF96. 2 cell line. PLoS ONE 9:e114333. doi: 10.1371/journal.pone.0114333

Pastorino, F., Brignole, C., Di Paolo, D., Nico, B., Pezzolo, A., Marimpietri, D., et al. (2006). Targeting liposomal chemotherapy via both tumor cell-specific and tumor vasculature-specific ligands potentiates therapeutic efficacy. Cancer Res. 66, 10073-10082. doi: 10.1158/0008-5472.can-06-2117

Pepper, M. S. (2001). Role of the matrix metalloproteinase and plasminogen activator-plasmin systems in angiogenesis. Arterioscler. Thromb. Vasc. Biol. 21, 1104-1117. doi: 10.1161/hq0701.093685

Perez-Tomas, R. (2006). Multidrug resistance: retrospect and prospects in anticancer drug treatment. Curr. Med. Chem. 13, 1859-1876. doi: 10.2174/ 092986706777585077

Prathipati, P., Dixit, A., and Saxena, A. K. (2007). Computer-aided drug design: integration of structure-based and ligand-based approaches in drug design. Curr. Comput. Aided Drug Des. 3, 133-148. doi: 10.2174/157340907780809516

Spector, A. A., and Yorek, M. A. (1985). Membrane lipid composition and cellular function. J. Lipid Res. 26, 1015-1035.

Stafford, J. H., and Thorpe, P. E. (2011). Increased exposure of phosphatidylethanolamine on the surface of tumor vascular endothelium. Neoplasia 13, 299-308. doi: 10.1593/neo.101366

Tian, Y., Jackson, P., Gunter, C., Wang, J., Rock, C. O., and Jackowski, S. (2006). Placental thrombosis and spontaneous fetal death in mice deficient in ethanolamine kinase 2. J. Biol. Chem. 281, 28438-28449. doi: 10.1074/jbc. m605861200

Troeira Henriques, S., Huang, Y. H., Chaousis, S., Wang, C. K., and Craik, D. J. (2014). Anticancer and toxic properties of cyclotides are dependent on phosphatidylethanolamine phospholipid targeting. Chembiochem 15, 1956-1965. doi: 10.1002/cbic.201402144

Vance, J. E. (2008). Thematic review series: glycerolipids. Phosphatidylserine and phosphatidylethanolamine in mammalian cells: two metabolically related aminophospholipids. J. Lipid Res. 49, 1377-1387. doi: 10.1194/jlr.r700020jlr200

Wang, C. K., Wacklin, H. P., and Craik, D. J. (2012). Cyclotides insert into lipid bilayers to form membrane pores and destabilize the membrane through hydrophobic and phosphoethanolamine-specific interactions. J. Biol. Chem. 287, 43884-43898. doi: 10.1074/jbc.m112.421198

Zhao, M. (2011). Lantibiotics as probes for phosphatidylethanolamine. Amino Acids 41, 1071-1079. doi: 10.1007/s00726-009-0386-9

Zhao, M., Li, Z., and Bugenhagen, S. (2008). 99mTc-labeled duramycin as a novel phosphatidylethanolamine-binding molecular probe. J. Nucl. Med. 49, 1345-1352. doi: 10.2967/jnumed.107.048603

Conflict of Interest Statement: The authors declare that the research was conducted in the absence of any commercial or financial relationships that could be construed as a potential conflict of interest.

The reviewer $\mathrm{CP}$ and handling Editor declared their shared affiliation, and the handling Editor states that the process nevertheless met the standards of a fair and objective review.

Copyright (c) 2017 Tan, Chan, Pusparajah, Lee, Chuah, Khan, Lee and Goh. This is an open-access article distributed under the terms of the Creative Commons Attribution License (CC BY). The use, distribution or reproduction in other forums is permitted, provided the original author(s) or licensor are credited and that the original publication in this journal is cited, in accordance with accepted academic practice. No use, distribution or reproduction is permitted which does not comply with these terms. 